\title{
Development of misfire detection algorithm using quantitative FDI performance analysis
}

\author{
Daniel Jung, Lars Eriksson, Erik Frisk and Mattias Krysander
}

\section{Linköping University Post Print}

\section{Tweet}

N.B.: When citing this work, cite the original article.

Original Publication:

Daniel Jung, Lars Eriksson, Erik Frisk and Mattias Krysander, Development of misfire detection algorithm using quantitative FDI performance analysis, 2015, Control Engineering Practice, (34), 49-60.

http://dx.doi.org/10.1016/j.conengprac.2014.10.001

Copyright: Elsevier

http://www.elsevier.com/

Postprint available at: Linköping University Electronic Press

http://urn.kb.se/resolve?urn=urn:nbn:se:liu:diva-114011 


\title{
Development of misfire detection algorithm using quantitative FDI performance analysis *
}

\author{
Daniel Jung *, Lars Eriksson, Erik Frisk, Mattias Krysander \\ Department of Electrical Engineering, Linköping University \\ SE-58183 Linköping, Sweden. \{daner,larer,frisk,matkr\}@isy.liu.se.
}

\begin{abstract}
A model-based misfire detection algorithm is proposed. The algorithm is able to detect misfires and identify the failing cylinder during different conditions, such as cylinder-to-cylinder variations, cold starts, and different engine behavior in different operating points. Also, a method is proposed for automatic tuning of the algorithm based on training data. The misfire detection algorithm is evaluated using data from several vehicles on the road and the results show that a low misclassification rate is achieved even during difficult conditions.
\end{abstract}

Key words: Misfire detection, Fault diagnosis, Fault detection and isolation, Kullback-Leibler divergence, Pattern recognition.

\section{Introduction}

Engine misfire detection is an important part of the OnBoard Diagnostics II (OBD-II) legislations to reduce exhaust emissions and avoid damage to the catalytic converter. Designing a misfire detection algorithm able to detect misfires during different conditions such as different operating points and cold starts requires tuning of many algorithm parameters. Manual tuning of these parameters in order to achieve satisfactory performance is often time-consuming.

The importance of having an accurate misfire detection algorithm requires a significant part of the available computational capacity. However, in order for the algorithm to be implementable in a vehicle the computational complexity of the algorithm must also be kept low. Thus, it is desirable to both reduce complexity of the algorithm and maintain sufficient detectability performance. In this work, the misfire detectability problem is analyzed and an automatically tuned misfire detection algorithm with low complexity is proposed.

An overview of misfire detection research can be found in [17] and the most common used signal for misfire de-

\footnotetext{
* The work is partially supported by Volvo Car Corporation and the Swedish Research Council within the Linnaeus Center CADICS.

* Corresponding author. Tel: +46 13 - 28 5743; fax: +46 13 - 139282
}

tection is the angular velocity measured at the flywheel. Signal analysis approaches used in, e.g., [19] and [20], utilize the oscillations in the angular velocity signal, related to the cylinder combustions, to detect when one or several cylinders fail to fire. In [24], a Markov chainbased algorithm is proposed to detect misfires. Compared to previous works, the angular velocity signal is used in this work to compute estimated torque which is used to detect misfires.

Beside the angular velocity signal, there are other measurements proposed for misfire detection, such as, engine vibration $[3,26,28]$ and ion current $[1,10,16]$. With respect to previous works which might require additional sensors, the angular velocity signal is used in this work since it is already available in modern vehicles.

A common model-based approach for misfire detection is to estimate the indicated torque to detect torque drops related to misfire $[4,14,30]$. In [4], indicated torque is estimated in the frequency domain and a metric of torque imbalance is used as a test quantity to detect misfires. However, in [4] only detection is considered and not identification of the misfiring cylinder. Other approaches considers estimating the cylinder pressures [18] or the relative changes in kinetic energy during compression and expansion stroke [29]. A contribution here with respect to previous works is a model-based misfire detection algorithm and an automatic off-line tuning strategy using training data of the misfire detection algorithm in order to handle different complicating conditions, such 
as cold starts.

The use of Kalman filters to estimate the indicated torque for misfire detection is proposed in $[12,14]$, and sliding mode observers in $[23,30,31]$. The use of observerbased solutions increases the computational complexity and limits the applicability in an on-line OBD system. Compared to these works, a contribution in this work is a model-based test quantity for misfire detection without the use of an observer to reduce computational complexity. The algorithm can handle different complicating conditions, such as cold starts. It is also able to identify the misfiring cylinder and can be automatically tuned off-line given training data.

The available maximum sampling resolution of the flywheel angular velocity signal is commonly $6^{\circ}$ which is used in [14], but also lower resolutions are used (e.g., $30^{\circ}[20]$ and $90^{\circ}$ [19]). High resolution data gives more information about each combustion but requires more computational power and is also more sensitive to measurement errors caused by flywheel manufacturing errors and signal sampling resolution [19]. In this work, measurements with an angular resolution of $30^{\circ}$ is used.

The misfire detection performance varies with different operating points, such as load and speed, and is also affected by disturbances such as: cycle-to-cycle and cylinder-to-cylinder variations, driveline oscillations, and flywheel manufacturing errors, see [19]. A contribution in this work is an analysis of measurement data, from different vehicles with the same type of engine, to see how misfire detection performance varies for different loads and speeds but also, in contrast to previous works, other complicating circumstances such as cold starts. The analysis is applied during the development process of the misfire detection algorithm to evaluate achieved performance for different designs of the algorithm.

The outline is as follows. First, the problem formulation is presented in Section 2. A model of the crankshaft is described in Section 3 and an analysis of misfire detectability performance in Section 4 . Then, the misfire detection algorithm is presented in Section 5 which is evaluated in Section 6. Finally, some conclusions are presented in Section 7 .

\section{Problem formulation}

Misfire detection is a difficult problem which is complicated by that the vehicle is operated in different conditions, such as different loads, speeds, cold starts, climate variations etc. The purpose of this work is to perform a quantitative analysis of the misfire detection performance, by analyzing measurement data, in order to develop a model-based misfire detection algorithm. The algorithm should be automatically tunable using training

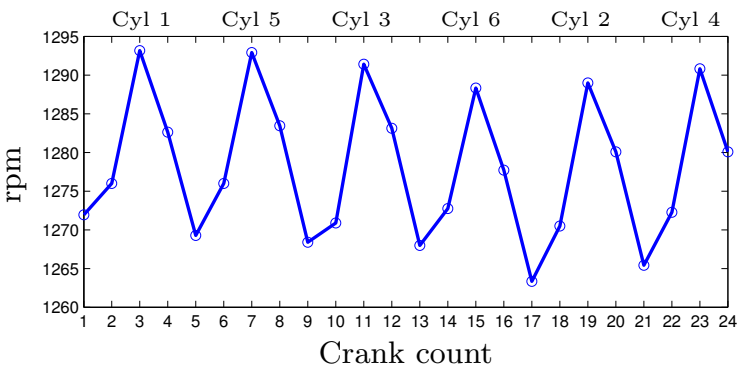

Fig. 1. Flywheel angular velocity where the crank counts associated to the combustion of each cylinder are shown.

data and should be able to handle variations in load and speed but also other conditions such as cold starts.

In this analysis, data from four cars with six-cylinder inline engines is used. The cylinders are numbered $1,2, \ldots, 6$ such that cylinder 1 is closest to the flywheel and driveline and cylinder 6 is located furthest away. A list of signals from the vehicle control system used in this work are shown in Table 1. The flywheel angular velocity $\omega$ is converted to rpm from the original signal which measures elapsed time between given angular intervals [8]. The air mass induced per revolution $m_{\mathrm{a}}$ $[\mathrm{g} / \mathrm{rev}]$ is used to represent the engine load [14].

The crank angle counter keeps track of the flywheel angle which is used to identify the firing cylinders during each revolution. With $30^{\circ}$ resolution, each cycle contains $2 \cdot 360^{\circ} / 30^{\circ}=24$ samples, i.e., 4 samples for each combustion in a six cylinder engine. The value of the crank angle counter identifies when during a cycle a sample is measured using the indexes $1,2, \ldots, 24$. The firing order of the engine is 1-5-3-6-2-4 and an example of samples related to each cylinder is shown in Fig. 1.

Table 1

A list of available signals.

\begin{tabular}{lcc}
\hline Signal & Variable & Unit \\
\hline Flywheel angular velocity & $\omega$ & $\mathrm{rpm}$ \\
Air mass induced per revolution & $m_{\mathrm{a}}$ & $\mathrm{g} / \mathrm{rev}$ \\
Crank angle counter & - & - \\
Catalyst warming flag & - & - \\
\hline
\end{tabular}

Misfire detection is considered based on the flywheel angular velocity signal with an angular sample resolution of $30^{\circ}$. Measurement data is available with intermittent misfires injected in all cylinders from four Volvo cars on road with the same type of six cylinder engine.

Two examples of the angular velocity signal with angular resolution of $30^{\circ}$ for two different operating points are shown in Fig. 2 and Fig. 3. In Fig. 2 data from low speed and low load is shown and each oscillation in the signal is related to a firing cylinder and a misfire is clearly visible as a larger speed drop in the signal. The angular velocity 


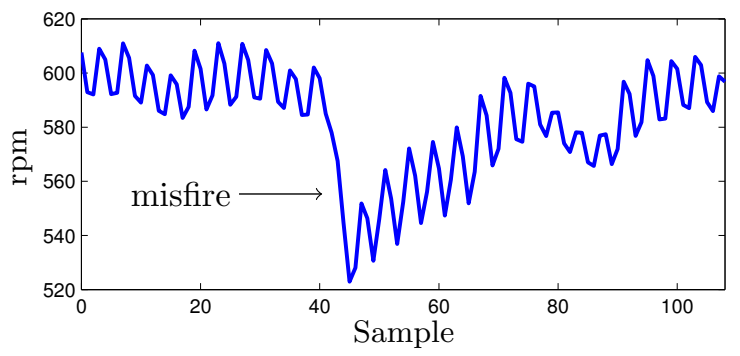

Fig. 2. Flywheel angular velocity measurements around speed $600 \mathrm{rpm}$ and load $0.33 \mathrm{~g} / \mathrm{rev}$.

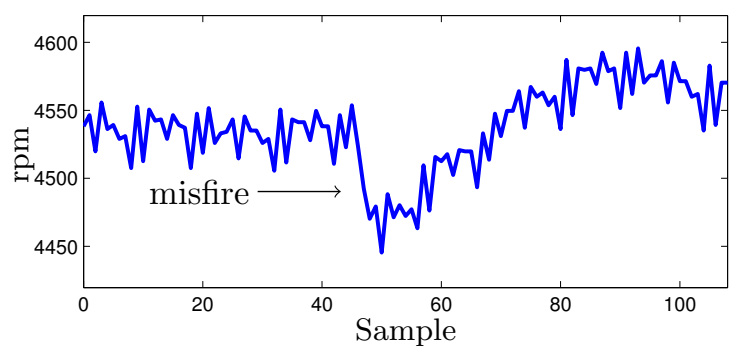

Fig. 3. Flywheel angular velocity measurements around speed $4500 \mathrm{rpm}$ and load $2.87 \mathrm{~g} / \mathrm{rev}$.

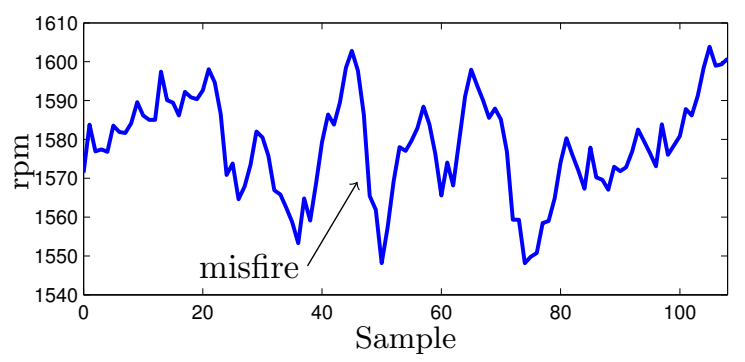

Fig. 4. Flywheel angular velocity measurements during cold start around speed $1580 \mathrm{rpm}$ and load $1.11 \mathrm{~g} / \mathrm{rev}$.

signal at high speed and load is shown in Fig. 3. A misfire is still visible as a larger speed drop but compared to the signal in Fig. 2, the oscillations in the signal are quite different. The shape of the signal related to each firing cylinder varies for different loads and speeds but also between the different cylinders. These variations must be taken into consideration in order to properly distinguish between fault-free combustions and misfires.

The vehicle control system uses a catalyst warming flag to indicate when there is a cold start. Detecting misfire during cold starts is difficult and an example of angular velocity measurements are shown in Fig. 4. Compared to the measurements in Fig. 2 and Fig. 3 it is more difficult to identify a misfire in the signal. Thus, misfire detectability performance during cold starts is important when designing a misfire detection algorithm.

Even if the misfires are easily identified manually in the flywheel signal in Fig. 2 and Fig. 3, implementing a misfire detection algorithm is difficult without first processing the signal. The trend of the flywheel signal follows the engine speed, thus selecting a threshold which adapts to varying speeds while distinguishing misfires from normal speed changes is difficult. Thus, the flywheel signal must be processed to easily distinguish misfires from normal combustions. In this work, a model-based approach is used to estimate the engine torque which will be discussed in the next section.

\section{Engine crankshaft model}

The previous section showed examples of when it was easy or difficult to manually detect a misfire in the angular velocity signal. A model-based approach to misfire detection is to estimate the indicated torque from the angular velocity signal. Models of varying complexity have been used to model the crankshaft, see for example, [8], [22], and [25]. However these models are computationally complex and are designed to captures other effects such as torsional vibrations of the crankshaft. Thus they are not usable for on-line misfire detection where the available computational power is limited. Reduction of the crankshaft model complexity is discussed in, for example, [21].

Simpler models describing the crankshaft are used in [4] and [14]. If torsional effects are ignored and the crankshaft is assumed to be stiff, the connection between the angular velocity and torques at the crankshaft can be described using Newton's second law as

$$
J \frac{d \omega}{d t}=\sum_{i=1}^{n_{\text {cyl }}} T_{\text {cyl }, i}-T_{\text {load }}-T_{\text {fr }}
$$

where $J$ is the moment of inertia, $\omega$ is crankshaft angular velocity, $T_{\mathrm{cyl}, i}$ is torque produced by cylinder $i, T_{\text {load }}$ is load torque, and $T_{\mathrm{fr}}$ is friction torque. The inertia $J$ usually depends on $\theta$ but is here assumed constant. The torque due to load $T_{\text {load }}$ is assumed to vary slowly compared to cylinder torque and the slow variation of friction $T_{\text {fr }}$ is included in $T_{\text {load }}$ [14].

The cylinder torque $T_{\mathrm{cyl}, i}$ represents the contributions from both the gas pressure force in cylinder $i$ and the moving mass of the piston and connecting rod. The contribution from the moving mass is a given function of the crankshaft angle and proportional to $\omega^{2}$ and can easily be compensated for, see equation (9) in [8]. In this work, this component is included in $T_{\mathrm{cyl}, i}$ since the effects are known and will be taken into consideration in the misfire detection algorithm. By considering the resulting cylinder torque

$$
T_{\mathrm{cyl}}=\sum_{i=1}^{n_{\mathrm{cyl}}} T_{\mathrm{cyl}, i}
$$


(1) can be written as

$$
J \frac{d \omega}{d t}=T_{\text {cyl }}-T_{\text {load }}
$$

Data is sampled synchronously with the crank angle. Therefore, (3) is expressed with crank angle as independent variable instead of time. The left hand side of (3) can then be expressed as

$$
\frac{d \omega}{d t}=\frac{d \omega}{d \theta} \frac{d \theta}{d t}=\frac{d \omega}{d \theta} \omega=\frac{1}{2} \frac{d\left(\omega^{2}\right)}{d \theta} .
$$

The torque on the right hand side of (3) can be estimated, for example, by using a Kalman filter [14]. In [14], the Kalman filter is motivated since data is sampled every $6^{\circ}$, and thus negative effects from flywheel manufacturing errors and quantification effects will result in a noisy signal. However, a Kalman filter estimator is computationally expensive and since the angular resolution here is $30^{\circ}$, the relative effects of the errors are much smaller and therefore negligible. Here, the derivative $\frac{d\left(\omega^{2}\right)}{d \theta}$ is approximated using Euler forward as

$$
J \frac{d \omega[\theta]}{d t}=J \frac{1}{2} \frac{d\left(\omega^{2}[\theta]\right)}{d \theta} \approx \frac{J}{2}\left(\frac{\omega^{2}[\theta+\Delta \theta]-\omega^{2}[\theta]}{\Delta \theta}\right) .
$$

The right hand side of (3) can be estimated from the angular velocity measurements by computing

$$
\frac{J}{2 \Delta \theta}\left(\omega^{2}[\theta+\Delta \theta]-\omega^{2}[\theta]\right)=T_{\text {cyl }}-T_{\text {load }}=T .
$$

Here, the parameter $J$ is unknown. Since it is the separation between data from misfires and fault-free combustions that is important, it is not important to have an accurate estimated torque $T$ as long as the same parameter value $J$ is used both during calibration and online running the test. Thus, instead of plotting the estimated torque in the following sections, a scaled estimated torque $T_{s}=\frac{T}{T_{\text {ref }}}$, where $T_{\text {ref }}$ is a selected reference torque, is used which is proportional to the estimated torque. The crank angle counts related to the estimated torque of each firing cylinder are shown in Fig. 5 using the same data as in Fig. 1.

\subsection{Misfire detection using estimated torque}

Two examples of estimated torque are shown in Fig. 6 and Fig. 7 where the torques are estimated given the flywheel data in Fig. 2 and Fig. 3 respectively. In Fig. 6, each firing cylinder is visible as a torque increase and the load and friction decreases the torque giving the oscillatory behavior. In both figures, a misfire is identified as a torque drop.

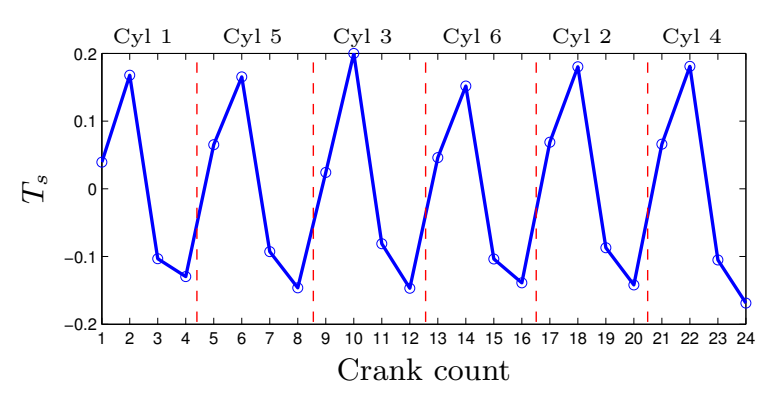

Fig. 5. Scaled estimated torque $T_{s}$ where the crank counts associated to the combustion of each cylinder are shown. Four samples are associated to each combustion.

In Fig. 6, each firing cylinder results in a torque increase and due to friction and load the torque falls between the firings. In Fig. 7, it is not as easy to distinguish the torque contributions from the individual cylinders. In the two examples, the estimated torque signals have a higher mean value for fault-free combustions compared to misfires. When comparing the estimated torque from several combustions of the same cylinder at similar speeds and loads, the trajectories are similar, even if they differ when comparing different cylinders. The trajectories are also similar when comparing data from different vehicles. This is important when calibrating a misfire detection algorithm since then it is not necessary to make separate calibrations for each individual vehicle.

To the eye it appears to be more difficult to detect misfires in the estimated torque signal compared to the original flywheel signal, but there are clear benefits of using the estimated torque. Estimated torques at similar speeds and loads have almost the same values, making it possible to compare measurements from different combustions. Whereas the flywheel signal follows the engine speed which makes comparisons between different combustions sensitive to the speed difference, especially during transients.

The variations in estimated torque between different cylinders can not be explained by the moving piston mass alone since then the estimated torque signal should look the same for all firing cylinders. Variations between different cylinders depending on their position was also observed in [20]. However, since the shape of the signal is always similar for each firing cylinder respectively, this can be compensated for when designing the test quantity by considering each cylinder separately.

It is assumed that $T_{\text {load }}$ varies much slower than the the variations of $T_{\text {cyl }}$. An example of computed mean torque is shown in Fig. 8, and it is clearly visible that the estimated mean torque varies from cycle-to-cycle. For the purpose of misfire detection, a misfire causes changes related to the fast oscillations and not the slowly moving trends in the estimated torque. Therefore, the mean torque for each cycle is subtracted from $T$. 


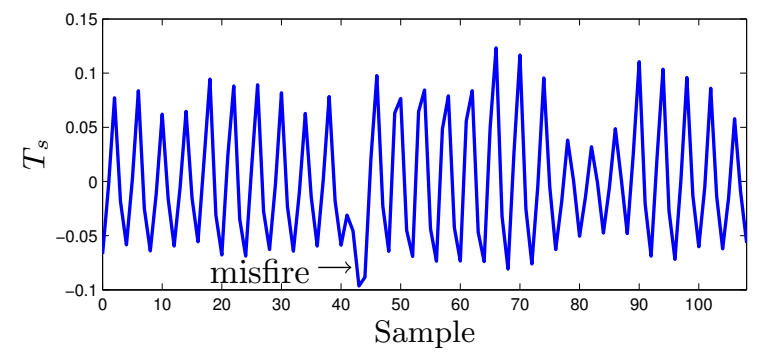

Fig. 6. Scaled estimated torque around speed $600 \mathrm{rpm}$ and load $0.33 \mathrm{~g} / \mathrm{rev}$.

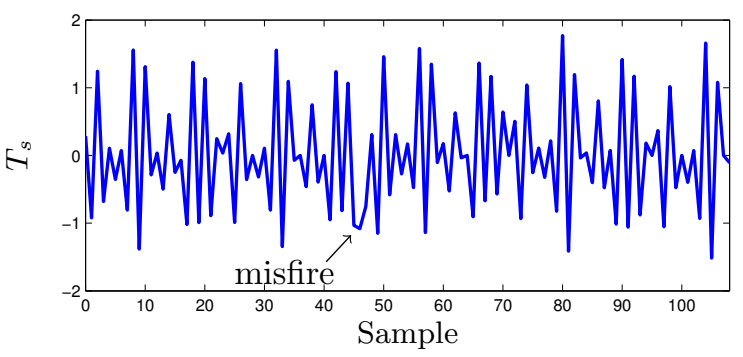

Fig. 7. Scaled estimated torque around speed $4500 \mathrm{rpm}$ and load $2.87 \mathrm{~g} /$ rev.

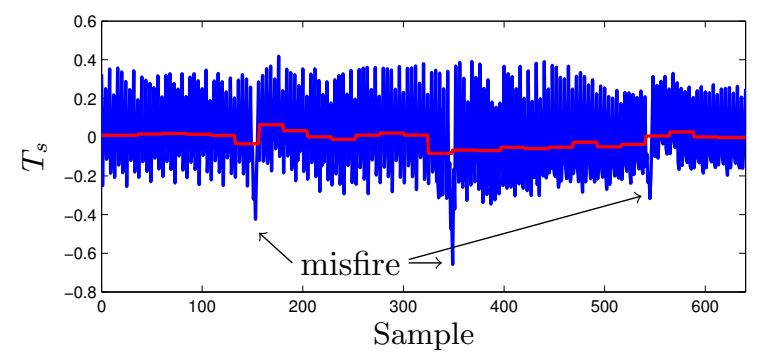

Fig. 8. Scaled estimated torque around speed $2200 \mathrm{rpm}$ and load $1.3 \mathrm{~g} / \mathrm{rev}$. The mean torque for each cycle is shown in the figure which is subtracted from the estimated torque.

\section{Misfire detectability analysis}

The shape of the estimated torque signal and misfire detection performance varies with speed and load which must be taken into consideration when designing a misfire detection algorithm. In this section, the behavior of the estimated torque signal during misfire is analyzed to see how signal characteristics varies for different samples, speeds, loads, but also during cold starts.

\subsection{Misfire visibility in data}

The number of data samples required for misfire detection in each cylinder depends on when and how long the effects of a misfire are visible in the flywheel angular velocity signal. An analysis of misfire visibility in different samples of the estimated torque signal, when the mean torque is subtracted, for different speed intervals has

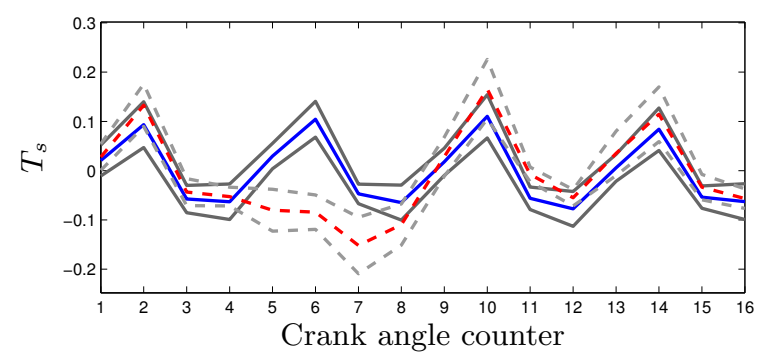

Fig. 9. Comparing scaled estimated torque in cycles with misfire in cylinder 5 (dashed line) with cycles without misfires (solid line) between 750-1500 rpm. The lines represent the mean torque and one standard deviation for each case.

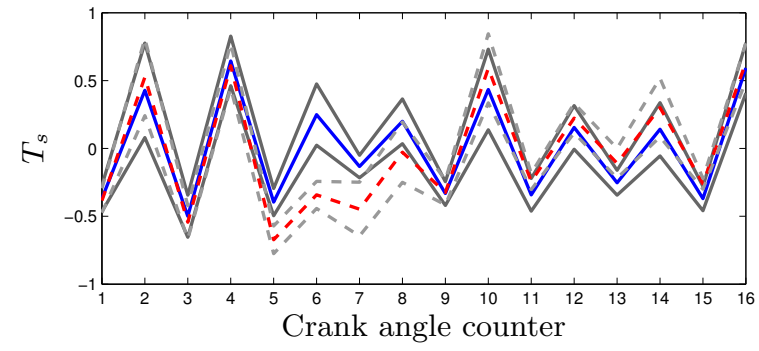

Fig. 10. Comparing scaled estimated torque in cycles with misfire in cylinder 5 (dashed line) with cycles without misfires (solid line) between 3000-3500 rpm.

been performed. Fig. 9 shows the estimated torque using measurements from a vehicle on the road in the angular velocity interval 750-1500 rpm and Fig. 10 in the interval 3000-3500 rpm. The figures compare different cycles with misfires in cylinder 5 with cycles with no misfires. The mean value of all estimated torques for each crank angle count and one standard deviation of the variations are plotted, where data without misfires are represented by the solid lines and misfires in cylinder 5 by dashed lines.

The deviation between fault-free data and misfire data is largest in the samples related to the combustion in cylinder 5 , i.e., crank angle counts $5-8$, see Fig. 5 . The misfire data is in average, slightly above the data of the fault-free cycles for the following combustions, i.e., crank angle counts 9-16 in the figure. However, the difference is relatively small for data during combustions after a misfire and will not be considered for misfire detection. The same results are present for different speeds, loads, and during cold starts which will be discussed more later.

\subsection{Stochastic representation of operating points}

In order to quantify misfire detection performance, a framework for representing the distributions of the estimated torque for different speeds and loads is useful. Here a stochastic representation of $T$ during misfires and in the fault-free case is presented similar to [9]. 
As concluded in the previous section, a misfire is visible in the data during the samples related to the misfiring cylinder. Therefore, for each cylinder we consider the estimated torque related to the cylinder as the vector $\mathbf{t}_{k}=\left(T_{j}, T_{j+1}, T_{j+2}, T_{j+3}\right)^{\mathrm{T}}$, where $j, j+1, j+2$, and $j+3$, correspond to the four crank counts of the firing cylinder $k$ as shown in Fig. 5, for example crank angle counts 5-8 for cylinder 5 . Then, let the distributions of $\mathbf{t}_{k}$ be described by the probability density functions (pdf) $q\left(\mathbf{t}_{k} \mid \omega, m_{a}\right)$ in the fault-free case and $p\left(\mathbf{t}_{k} \mid \omega, m_{a}\right)$ in the misfire case which both depend on speed $\omega$ and load $m_{a}$.

Given the pdfs $q\left(\mathbf{t}_{k} \mid \omega, m_{a}\right)$ and $p\left(\mathbf{t}_{k} \mid \omega, m_{a}\right)$ representing fault-free data and misfire data respectively for cylinder $k$, the misfire detection performance given $\mathbf{t}_{k}$ is defined by how separated the two distributions are for different speeds, loads, and cylinders. A measure for quantifying this separation will be discussed next.

\subsection{Kullback-Leibler divergence}

Fault-free data and misfire data are drawn from two distributions which are mainly functions of speed, load, and cylinder, as discussed in the previous section. A measure of how easy it is to detect a misfire is to quantify the separation, or difference, between the probability density functions in the misfire case and the fault-free case. Mainly, the interesting question is to quantify how easy is it to detect a misfire and one way to measure this is to use the Kullback-Leibler divergence [15].

The Kullback-Leibler divergence from a pdf $p$ to another pdf $q$ can be written as

$$
K(p \| q)=E_{p}\left[\log \frac{p}{q}\right]
$$

i.e, it is the mean value of the log-likelihood ratio given that $p$ is the true distribution. $K(p \| q)$ is non-negative and equal to zero if and only if $p=q$. It is also required that $q$ is non-zero, in the support of $p$.

Let $p$ and $q$ be the probability density functions of the estimated indicated torque such that $p$ represents the pdf during misfire and $q$ the pdf of fault-free combustions. Then, $K(p \| q)$ can be interpreted as a quantified measure of how difficult it is to detect a misfire [7].

In cases where $p$ and $q$ are well separated, the loglikelihood ratio in (7) depend on the tail of $q$. Often, knowledge about the tails of the pdf is poor when using data to estimate the distributions due to few samples in the tails. Also, if the probability of $q$ is close to zero when $p$ is large, it is difficult to numerically estimate (7). Finding an approximative parametric distribution based on the data such that (7) can be solved explicitly is a solution to this problem.

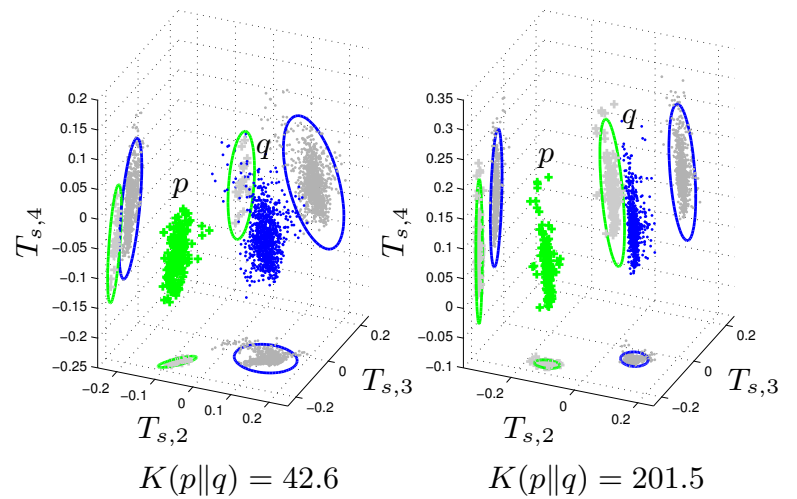

Fig. 11. Two examples of computed Kullback-Leibler divergence from misfire data (plus) to fault-free data (dot), from operating points with high and low Kullback-Leibler divergence. 3- $\sigma$ ellipses are plotted for each $2 \mathrm{D}$ projection of the data.

If $p \sim \mathcal{N}\left(\mu_{p}, \boldsymbol{\Sigma}_{p}\right)$ and $q \sim \mathcal{N}\left(\mu_{q}, \boldsymbol{\Sigma}_{q}\right)$ are $k$-dimensional multivariate Gaussian distributed, (7) can be computed analytically as

$$
\begin{aligned}
K(p \| q) & =\frac{1}{2}\left(\operatorname{tr}\left(\boldsymbol{\Sigma}_{q}^{-1} \boldsymbol{\Sigma}_{p}\right)+\left(\mu_{q}-\mu_{p}\right)^{\mathrm{T}} \boldsymbol{\Sigma}_{q}^{-1}\left(\mu_{q}-\mu_{p}\right)\right. \\
& \left.-k-\log \left(\frac{\operatorname{det} \boldsymbol{\Sigma}_{p}}{\operatorname{det} \boldsymbol{\Sigma}_{q}}\right)\right) .
\end{aligned}
$$

Two examples of how fault-free data and misfire data are distributed and the corresponding calculated KullbackLeibler divergences are shown in Fig. 11. Since $\mathbf{t}_{k}$ is four dimensional $\left(T_{1}, T_{2}, T_{3}, T_{4}\right)$ and can not be directly illustrated, only the last three samples are plotted. The distributions of fault-free data and misfire data of $\mathbf{t}_{k}$ are approximated as multivariate Gaussian distributions, where the mean values and covariance matrices are estimated from the data, and the Kullback-Leibler divergence is calculated using (8). To visualize the approximated Gaussian distributions of the data, ellipses representing three standard deviations $(3-\sigma)$ are plotted for each $2 \mathrm{D}$ projection of the data. A higher KullbackLeibler divergence represents more separated distributions which makes it easier to distinguish a misfire from fault-free data.

\subsection{Misfire detectability performance for different op- erating points}

Before developing a misfire detection algorithm, the misfire detection performance is here evaluated as a function of load, speed, and cylinder, by analyzing the difference between the distributions of fault-free data and misfire data. If the data from the two cases are well separated, it should be easy to design a test quantity able to make correct classifications whether there is a misfire or not. This information is useful later when designing the test 
quantity since it will show, for example that data from different cylinders should be considered separately and that it is more difficult during low speeds to distinguish misfires from fault-free data than for higher speeds. Another interesting application during the design of the test quantity is that the Kullback-Leibler divergence can be used to motivate design choices that improve detection performance. For example, it is shown later that when normalizing the estimated torque with air mass flow the detection performance increases.

As expected in Section 4.1, a misfire is mostly visible in the data samples related to the misfiring cylinder. Therefore, different crank angle count intervals will be used for misfire detection in each cylinder, i.e., detection and identification of the misfiring cylinder can be performed simultaneously.

The following analysis shows how misfire detection performance varies with different speeds, loads, and cylinders. The estimated torque is associated to each cylinder based on the crank counts as shown in Fig. 5. Data measured at similar speeds and loads has similar values, see Fig. 11. To analyze the separation between misfire data and fault-free data, the pdfs $p\left(\mathbf{t}_{k} \mid \omega, m_{a}\right)$ and $q\left(\mathbf{t}_{k} \mid \omega, m_{a}\right)$ are assumed multivariate Gaussian distributed where mean and covariances are estimated using data from real driving scenarios. Thus, the Kullback-Leibler divergence can be calculated using (8).

Here, the pdfs for each speed $\omega$, load $m_{a}$, and cylinder, are estimated using data, from all four vehicles on the road, selected from intervals $[\omega-250, \omega+250]$ and $\left[m_{a}-0.2, m_{a}+0.2\right]$ where there are at least 100 samples available from fault-free data and misfire data respectively. The result of the detectability analysis of cylinder 1 is shown in Fig. 12. The figure shows the computed Kullback-Leibler divergence for different speeds and loads and lies in the interval 31.4 - 194.1. Also, the results varies for different cylinders. The result for cylinder six is shown in Fig. 13 where overall Kullback-Leibler divergence is generally lower compared to cylinder 1 (in the interval 34.9 - 93.6) indicating worse detection performance. This indicates that for good misfire detection performance, a misfire detection algorithm needs to consider each cylinder individually since the data varies between the cylinders.

Comparing the results for all cylinders show that detectability performance of cylinders 1,3 , and 5 have similar shape as in Fig. 12 and that cylinders 2, 4, and 6 resemble Fig. 13. Still the result indicates that it is, in general, more difficult to detect misfires at low speeds and loads and easier at high speeds and loads. Note that the Kullback-Leibler divergence increases mainly due to higher speeds for cylinder 1 . Both higher speed and load increases the Kullback-Leibler divergence for cylinder 6 but not as much as for cylinder 1 .

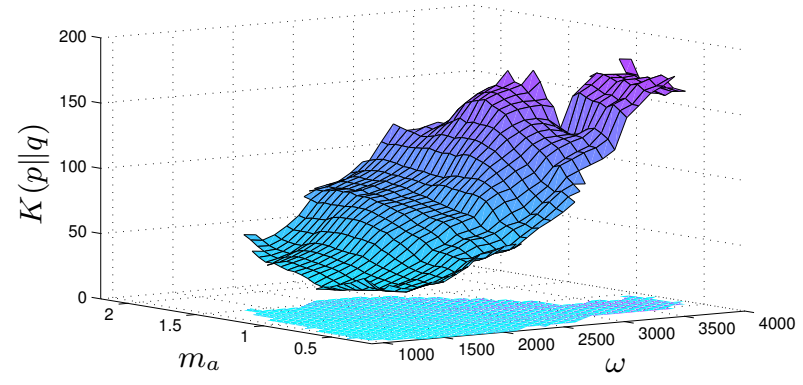

Fig. 12. Misfire detectability performance given $\mathbf{t}_{1}$ for cylinder 1 quantified using Kullback-Leibler divergence as a function of load and speed.

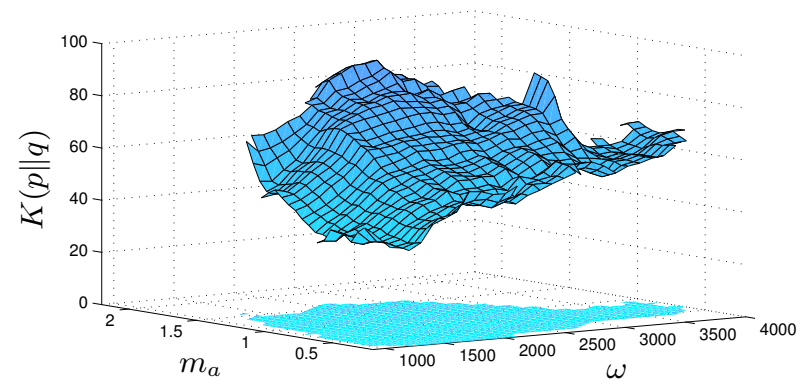

Fig. 13. Misfire detectability performance given $\mathbf{t}_{6}$ for cylinder 6 quantified using Kullback-Leibler divergence as a function of load and speed.

Note that by changing the data intervals in the analysis, the corresponding graphs as in Fig. 12 and Fig. 13 can be modified. Narrower intervals result in higher Kullback-Leibler divergence and larger intervals reduce the Kullback-Leibler divergence. This analysis is used to balance misfire detection performance and the number of operating points describing different loads and speeds depending on algorithm requirements.

To visualize how the shape of the estimated torque varies with load and speed, samples from three of four crank angle counts are plotted against each other. The data set is from a vehicle on the road and covers different speeds and loads. The estimated torque related to cylinder 5 have crank angle counts 5-8 where the estimated torque at the crank angle counts $6-8$ are plotted against each other. Fig. 14 and Fig. 15 show how the estimate torque change with different loads and speeds respectively using the same set of data. Darker colors represent higher loads in Fig. 14 and higher speeds in Fig. 15. Fault-free data is represented by dots and misfire data by stars. To clarify the separation between fault-free data and misfire data, a dashed line is placed in the figure to show the separation.

Fig. 14 shows that the distance between fault-free data and misfire data increases with higher loads since the color intensifies, the longer from the dashed line a data point is positioned. With increasing speed, the average 


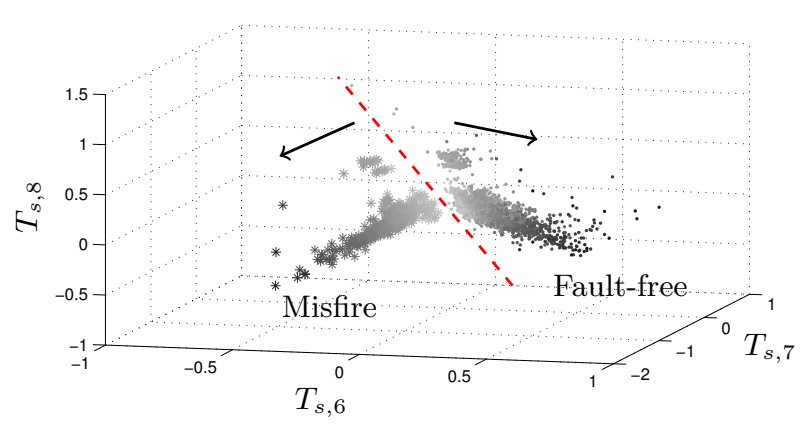

Fig. 14. Scaled estimated torque from cylinder 5 where darker colors represent higher loads. The dashed line is used to show how fault-free data and misfire data are separated. The arrows represent the direction of increasing load.

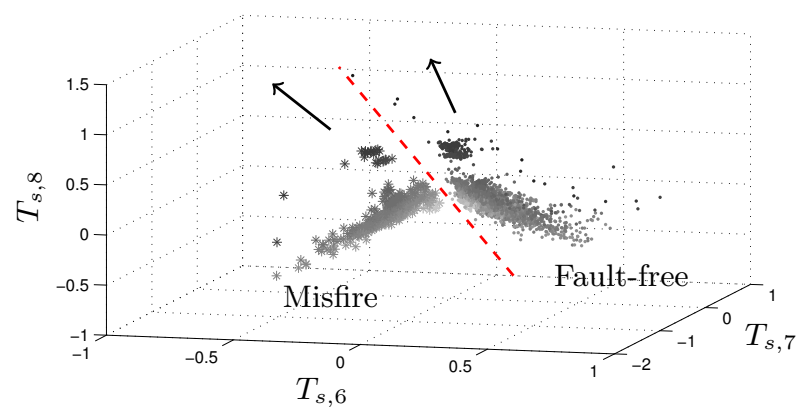

Fig. 15. Scaled estimated torque from cylinder 5 where darker colors represent higher speeds. The dashed line is used to show how fault-free data and misfire data are separated. The arrows represent the direction of increasing speed.

distance from the dashed line is not changed but instead where the data is located. This indicates that the change of the shape of the estimated torque as was shown when comparing Fig. 6 and Fig. 7 is mainly depending on speed. However, if looking at the distributions of the measurements within a given speed interval when varying the load or vice versa, it is not necessary that increased load results in more separated data than increased speed. The covariance of the data for different speeds and loads is also important to measure how difficult it is to distinguish misfires from fault-free combustions. It is difficult to see how much data is spread out for different loads and speeds in Fig. 14 and Fig. 15 respectively, but this is taken into consideration in Fig 12 and Fig 13.

\subsection{Cold starts}

A cold start occurs when the car has not been used for a while and the catalytic converter is cold. The catalyst is ineffective at low temperatures and must therefore be heated before functioning $[13,27]$. The heating of the catalytic converter is made by controlled late ignitions in the cylinders to release more heat in the exhaust gases. Also the cold cylinder walls will affect the combustion

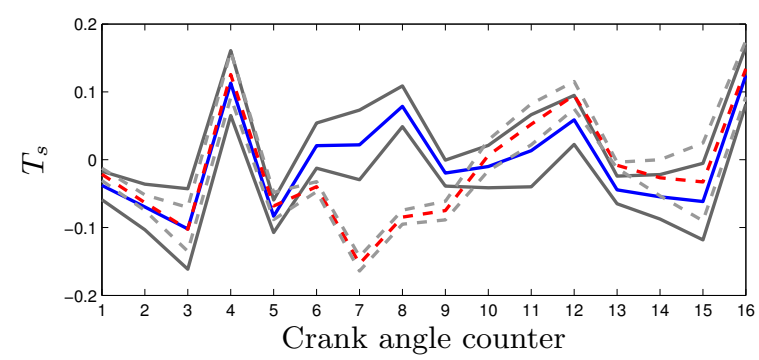

Fig. 16. Comparing scaled estimated torque in cycles with misfire in cylinder 5 (dashed line) with cycles without misfires (solid line) during cold start. The lines represents the mean torque and one standard deviation for each case.

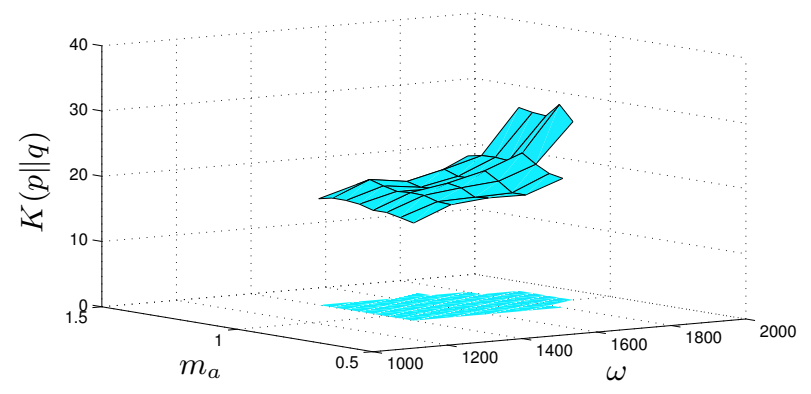

Fig. 17. Misfire detectability performance given $\mathbf{t}_{1}$ for cylinder 1 during cold starts quantified using Kullback-Leibler divergence as a function of speed and load.

variations which complicates the misfire detection.

Since a later ignition is induced during cold starts, the effects of a misfire are observable later in the measurements as shown in Fig. 16. Misfires during cold starts are visible during crank angle counts 6-9, see Fig. 16, instead of 5-8 as during normal driving, see Fig. 9.

An evaluation of misfire detection performance using the Kullback-Leibler divergence as in the previous section is shown in Fig. 17 and Fig. 18 for cylinder 1 and 6 respectively. However, due to a limited set of data, the number of samples required before estimating the KullbackLeibler divergence at a specific speed and load is chosen as 50 instead of 100 . The figures show that since cold starts occur during start up of the engine, data is only available at low speeds and loads. The Kullback-Leibler divergence lies in the interval 15.0 - 29.6 for cylinder 1 and in the interval 14.1 - 19.0 for cylinder 6 which is lower than for the same speeds and loads in the normal case.

As shown in Fig. 16, misfires during cold starts are visible at later crank angle counts since the cylinders fires at a later crank angle. Therefore in Fig. 19, the estimated torque from crank angle counts 7,8 , and 9 are plotted against each other where a darker color represents higher speed. The figure shows a comparison of fault-free and misfire data during cold starts. It is seen that the separation between fault-free and misfire case decreases during 


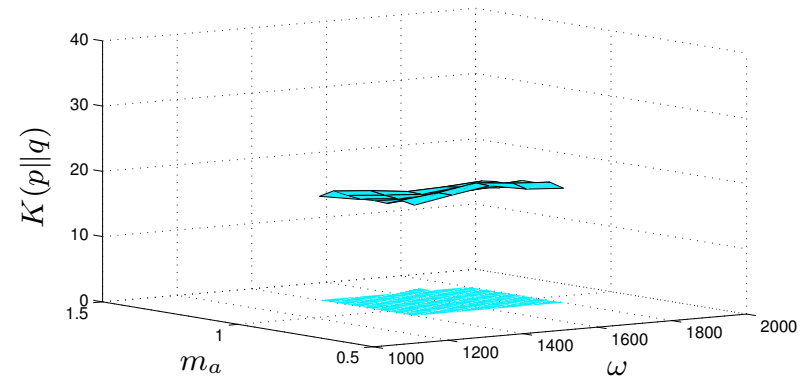

Fig. 18. Misfire detectability performance given $\mathbf{t}_{6}$ for cylinder 6 during cold starts quantified using Kullback-Leibler divergence as a function of load and speed.

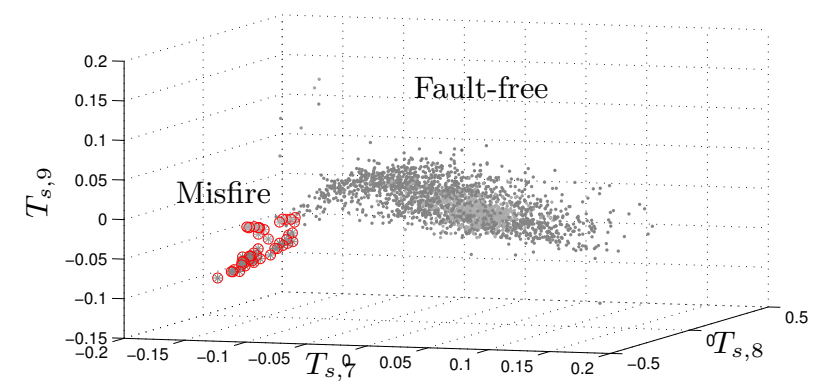

Fig. 19. Scaled estimated torque $\mathbf{t}_{5}$ during cold starts from cylinder 5 where darker colors represents higher speeds is plotted. Misfire data is marked by circles.

cold starts compared to normal driving. However, faultfree data and misfire data are fairly separated and thus it is possible to distinguish them from each other with few misclassifications.

\section{Design of misfire detection algorithm}

Here, the result from the analysis in the previous section is used to design a misfire detection algorithm. The analyses showed how misfire data is separated from faultfree data and how this separation varies with different speeds, loads and during cold starts. The distance between fault-free data and misfire data is mainly correlated with load while speed changes the torque trajectory. By categorizing the estimated torque based on speed, load, and cylinder, data which are similar to each other can be compared in order to detect misfires. If the variation related to load is compensated for, the number of modes to partition measurement data into, in the misfire detection algorithm, can be reduced.

\subsection{Normalization with respect to engine load}

The intake manifold pressure signal is not available in this work. An approximative relation between the air mass induced per revolution and the intake manifold

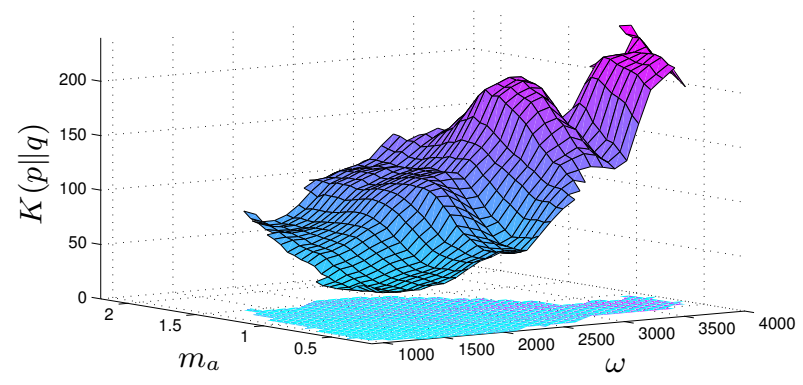

Fig. 20. Misfire detectability performance given $\mathbf{t}_{1}$ normalized with engine load for cylinder 1 quantified using Kullback-Leibler divergence as a function of load and speed.

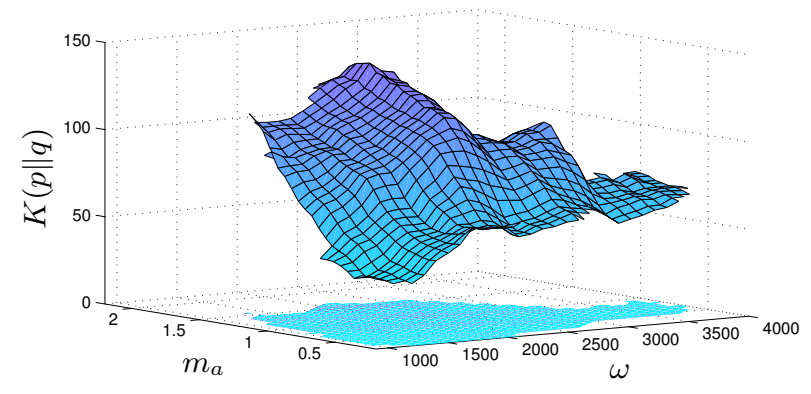

Fig. 21. Misfire detectability performance given $\mathbf{t}_{6}$ normalized with engine load for cylinder 6 quantified using Kullback-Leibler divergence as a function of load and speed.

pressure is found using the volumetric efficiency $\eta_{\mathrm{v}}$, defined in [13] as

$$
\eta_{\mathrm{v}}=\frac{m_{\mathrm{a}} / 2}{\rho_{\mathrm{a}, i} V_{\mathrm{d}}}
$$

where $m_{\mathrm{a}}$ is the air mass induced per cycle, $\rho_{\mathrm{a}, i}$ is the inlet air density, and $V_{\mathrm{d}}$ is the displacement volume. The ideal gas law, $p_{\mathrm{im}}=\rho_{\mathrm{a}, i} R \mathcal{T}$, where $R$ is the specific gas constant and $\mathcal{T}$ is absolute temperature of the inlet air, gives that

$$
\eta_{\mathrm{v}}=\frac{m_{\mathrm{a}}}{2 \rho_{\mathrm{a}, i} V_{\mathrm{d}}}=\frac{m_{a} R \mathcal{T}}{2 p_{\mathrm{im}} V_{\mathrm{d}}} \Leftrightarrow p_{\mathrm{im}}=m_{\mathrm{a}} \frac{R \mathcal{T}}{2 V_{\mathrm{d}} \eta_{\mathrm{v}}} .
$$

Assuming that the term $\frac{R \mathcal{T}}{2 V_{\mathrm{d}} \eta_{\mathrm{v}}}$ is constant then $p_{\mathrm{im}} \propto$ $m_{\mathrm{a}}$. Then, the estimated torque (6) is normalized by $m_{\mathrm{a}}$ to get a quantity which is not dependent on load.

Again, the Kullback-Leibler divergence is calculated to compare how the misfire detection performance varies for different speeds and loads when normalizing the estimated torque with $m_{\mathrm{a}}$. The results are shown in Fig. 20 and Fig. 21 for cylinder 1 and 6 respectively, it is seen that the Kullback-Leibler divergence has increased for all speeds and loads compared to before normalization. The Kullback-Leibler divergence of cylinder 1 lies in the interval 41.3 - 252.0 and cylinder 6 lies in the interval $35.6-140.6$. 


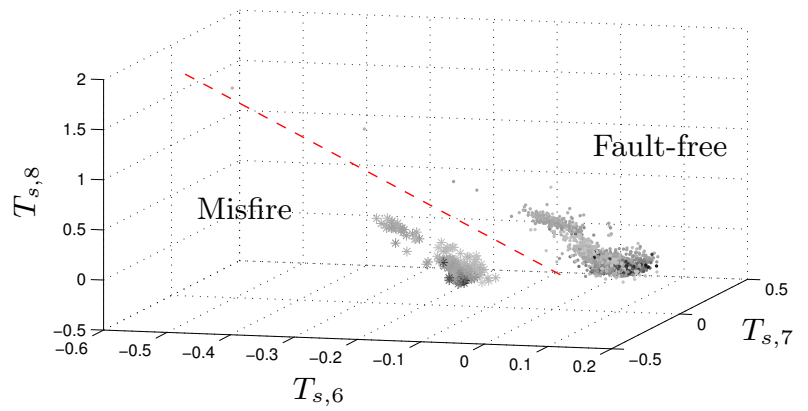

Fig. 22. Scaled estimated torque, normalized with respect to air mass flow, from cylinder 5 where darker colors represent higher loads.

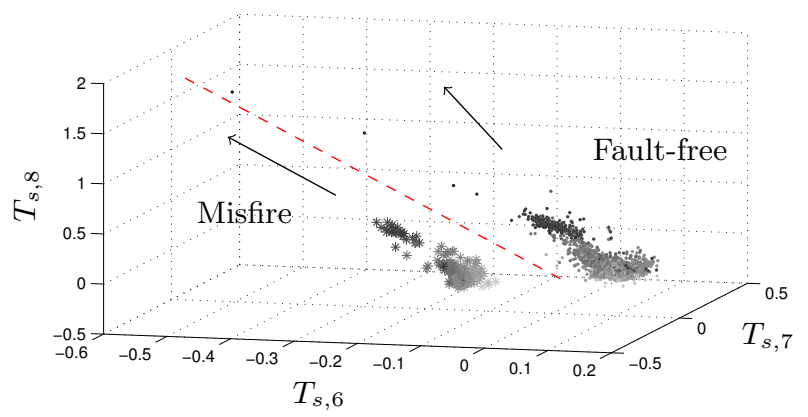

Fig. 23. Scaled estimated torque, normalized with respect to air mass flow, from cylinder five where darker colors represent higher speeds.

Data of normalized estimated torque is plotted in Fig. 22 and Fig. 23 where darker points represent increasing load and speed respectively. Compared to Fig. 14 and Fig. 15, the variations of the data points with respect to different loads is reduced. The variation of the estimated torque related to speed will be taken into consideration by comparing data in different speed intervals.

Fig. 24 shows normalized torque from cold starts and highlights that the separation between the fault-free data and misfire data is still smaller than for normal combustions but the distributions are fairly well separated. The computed Kullback-Leibler divergence for cylinder 1 and 6 lies in the intervals 15.6 - 30.1 and 14.7 - 19.0 and are slightly better than without normalizing the torque signal.

\subsection{Detecting misfires}

Commonly in model-based approaches to fault detection, redundancy of the system model and available sensors is utilized to detect inconsistency between the observations and the model [6]. However, since the information about the combustions here is only available via the estimated torque signal, some type of pattern recognition is necessary to distinguish a misfire from a faultfree combustion, see [2] and [11].

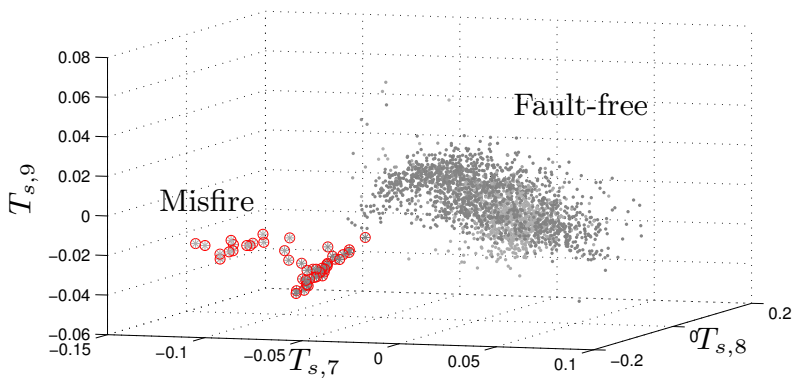

Fig. 24. Scaled estimated torque, normalized with air mass flow, from fault-free combustions and misfires during cold starts where darker colors represents higher speeds.

The data in Fig. 22 and Fig. 23, and also in Fig. 24, indicates that fault-free data and misfire data in general are well separated. Thus, it is possible to use a linear classification strategy to separate the fault-free data and misfire data with few misclassifications.

A misfire is visible in the four samples of estimated torque related to the misfiring cylinder. Here, the test quantity $r$ is designed by weighting all samples for each combustion such that data from a misfire is maximally separated from data of a fault-free combustion. Let $\mathbf{w}_{k}=\left(w_{1}, w_{2}, w_{3}, w_{4}\right)^{\mathrm{T}}$ be the weights for a given speed and cylinder $k$ and $\mathbf{t}_{k}=\left(T_{j}, T_{j+1}, T_{j+2}, T_{j+3}\right)^{\mathrm{T}}$ is the normalized estimated torque from cylinder $k$ where the crank angle counts $j, j+1, j+2$, and $j+3$ for each cylinder are given in Table 2 . Then, the test quantity $r$ is given by

$$
r=\mathbf{w}_{k}^{\mathrm{T}} \mathbf{t}_{k}+\beta
$$

where $\beta$ is a constant, depending on speed and cylinder, such that $r<0$ when there is a misfire and $r>0$ in the fault-free case.

Thus, the goal is to find the weights $\mathbf{w}_{k}$ such that the pdf of misfire data $p\left(\mathbf{w}_{k}^{\mathrm{T}} \mathbf{t}_{k}\right)$ are maximally separated from fault-free data $q\left(\mathbf{w}_{k}^{\mathrm{T}} \mathbf{t}_{k}\right)$. Here, Support Vector Machines (SVM) is used to find the weights $w$.

\subsection{Support Vector Machines}

Support Vector Machines (SVM) is a machine learning approach that can be used for data classification $[2,5]$. The method utilizes samples from training data, called support vectors, to find the maximum margin between the different classes of data. A threshold for the classification is then selected within this margin. The advantage of using SVM is that the problem of finding optimal weights $w$ that maximizes the margin is a convex optimization problem and thus a global optimum can easily be found.

Note that SVM uses no information about the rest of the training data except the support vectors. Thus, it is 
Table 2

The crank counts of the samples to be weighted in (11) for each cylinder used for misfire detection during normal driving and cold starts.

\begin{tabular}{ccc}
\hline Cylinder & Normal & $\begin{array}{c}\text { Cold start/ } \\
\text { catalyst warming }\end{array}$ \\
\hline 1 & $1,2,3,4$ & $2,3,4,5$ \\
2 & $17,18,19,20$ & $18,19,20,21$ \\
3 & $9,10,11,12$ & $10,11,12,13$ \\
4 & $21,22,23,24$ & $22,23,24,1$ \\
5 & $5,6,7,8$ & $6,7,8,9$ \\
6 & $13,14,15,16$ & $14,15,16,17$ \\
\hline
\end{tabular}

more important that the available data is representative of the whole operating range of the vehicle rather than having lots of training data. However, the method is sensitive to outliers and faulty classified data. In cases where the different classes of data in the training data set are not separable from each other the misclassified samples are penalized in the optimization.

\subsection{Misfire detection algorithm design summary}

From the analysis in Section 4 it was shown that misfires during cold starts are visible at later crank angle counts compared to normal driving. Therefore based on the status of the catalyst warming flag, different samples are used by the misfire detection algorithm. The samples $\mathbf{t}_{k}$ weighted together in (11) for each cylinder $k$ during normal driving and cold starts are shown in Table 2.

A summary of the off-line design procedure of the misfire detection algorithm is presented here in the following steps.

(1) Use training data, covering all speeds and loads in the operating range, to compute estimated torque $T$ and subtract the mean estimated torque for each cycle, then normalize it with $m_{\mathrm{a}}$.

(2) Select the crank angle counts of the estimated torque for each cylinder which will be used to separate misfires from fault-free combustions as discussed in Section 3.

(3) Select speed intervals such that misfire data $p$ is well separated from fault-free data $q$, i.e., the KullbackLeibler divergence is sufficiently high for each interval.

(4) For each speed interval, cylinder, and cold start, estimate a vector $\mathbf{w}_{k}$ such that misfire data and fault-free data have a maximum margin using SVM.

(5) Select constants $\beta$ for each interval balancing the criteria for false-alarm rate and missed detection rate. Increasing $\beta$ will reduce the false alarm rate while decreasing it will reduce the missed detection rate.
Table 3

Training data during normal driving for automatic tuning of misfire detection algorithm.

\begin{tabular}{cccc}
\hline Vehicle & Data set & Fault-free & Misfire \\
\hline 1 & 1 & 98923 & 2066 \\
2 & 2 & 10680 & 224 \\
2 & 3 & 20551 & 435 \\
\hline
\end{tabular}

Table 4

Training data during cold starts for automatic tuning of misfire detection algorithm.

\begin{tabular}{cccc}
\hline Vehicle & Data set & Fault-free & Misfire \\
\hline 3 & 9 & 6121 & 127 \\
3 & 10 & 3971 & 82 \\
4 & 11 & 2502 & 52 \\
\hline
\end{tabular}

To find good parameters $\mathbf{w}_{k}$ and $\beta$ in (11) such that the misclassification rate of $r$ is minimized, it is necessary to have measurements covering the whole operating range of the vehicle. Also, collecting data from a couple of different vehicles is useful to capture the variations between different vehicles.

\section{Evaluation}

In this evaluation, the misfire detection algorithm (11) is automatically designed using the steps in Section 5.4 where weights and thresholds are optimized using training data. The misfire detection algorithm is then evaluated on other driving data sets which contains both urban and highway driving. Intermittent misfires have been injected in all cylinders and it is assumed that no other misfires have occurred in the data sets. A list of the training data for normal driving and cold starts are shown in Tables 3 and 4 . The tables show the number of fault-free combustions and misfires in each data set. The test quantity is evaluated on a number of driving scenarios from four vehicles with the same type of six cylinder engine. All cars have not been used to collect the same types of measurements, for example, there is not cold start data from all cars.

The parameters $\mathbf{w}_{k}$ and $\beta$ for different speeds and cylinders are optimized using SVM in Matlab. The thresholds are trade-offs between false alarms and missed detections, i.e., the number of misclassifications. Here, the thresholds are given by the SVM optimization algorithm, svmtrain, in Matlab.

Data is associated to the closest operating point in the algorithm which are here selected as the following speeds: $500 \mathrm{rpm}, 1000 \mathrm{rpm}, 1500 \mathrm{rpm}, 2000 \mathrm{rpm}$, $2500 \mathrm{rpm}, 3000 \mathrm{rpm}, 3500 \mathrm{rpm}, 4000 \mathrm{rpm}$, and $4500 \mathrm{rpm}$. Thus, weights and thresholds are optimized for cylinders $*$ speed intervals $=54$ operating points. Cold starts occurs here at a limited 


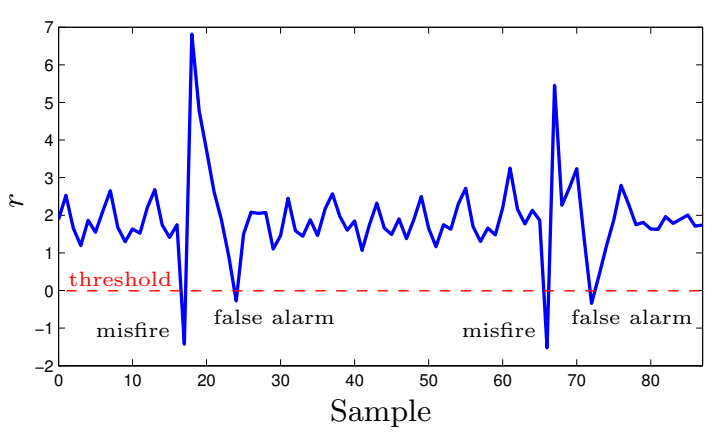

Fig. 25. In data set 7 where parameters are optimized using SVM, the oscillations in the test quantity after some misfires goes below the threshold and result in false alarms.

range of engine speeds and requires an additional cylinders $*$ speed intervals $=6 \cdot 2=12$ operating points.

\subsection{Misfire detection}

First, the focus will be on data from normal driving and later the focus will be on data from cold starts. The results from the evaluation when using SVM are shown in Table 5. The data shows the number of fault-free combustions and misfires for each data set. The result of the misfire detection algorithm is presented in percent of how many of the misfires that was not detected (MD) and how many of the fault-free combustions that caused false alarms (FA) for each data set. The evaluations show good results where the number of false alarms and missed detections are low except for data set 7 which have more false alarms compared to the other data sets. The false alarms for data set 7 are caused by torsional vibrations in the crankshaft and driveline right after a real misfire causing an oscillating test quantity to go below zero, see Fig. 25. In this case, the lumped crankshaft assumption has problem to handle the torsional vibrations. An approach could be to pre-filter the flywheel signal using results from [21].

Histograms of $r$ for data sets 6 and 7 in Table 5 are shown in Fig. 26 and Fig. 27 respectively. Data from all cylinders and speeds are included in the histograms and the threshold is represented by the dashed line where samples to the left are classified as misfires. Data set 6 had neither false alarms nor missed detections and the histograms of $r$ are shown in Fig. 26. Data set 7 had the most false alarms of the evaluation sets and the distribution of $r$ is shown in Fig. 27. In both cases, the distributions of fault-free data and misfire data are well separated from each other which indicates that the misfire detection algorithm should be robust to small variations of the distributions.

The results when evaluating the performance on data from cold starts are shown in Table 6 . Even if cold starts are considered to be more difficult to detect misfires the
Table 5

Evaluation of misfire algorithm during normal driving.

\begin{tabular}{cccccc}
\hline Vehicle & Set & Fault-free & Misfire & FA & MD \\
\hline 1 & 4 & 41444 & 863 & $0.0048 \%$ & $0.23 \%$ \\
1 & 5 & 93251 & 1941 & $0 \%$ & $0 \%$ \\
2 & 6 & 45496 & 949 & $0 \%$ & $0 \%$ \\
2 & 7 & 48881 & 1013 & $0.090 \%$ & $0 \%$ \\
3 & 8 & 49218 & 1020 & $0.012 \%$ & $0 \%$ \\
\hline
\end{tabular}

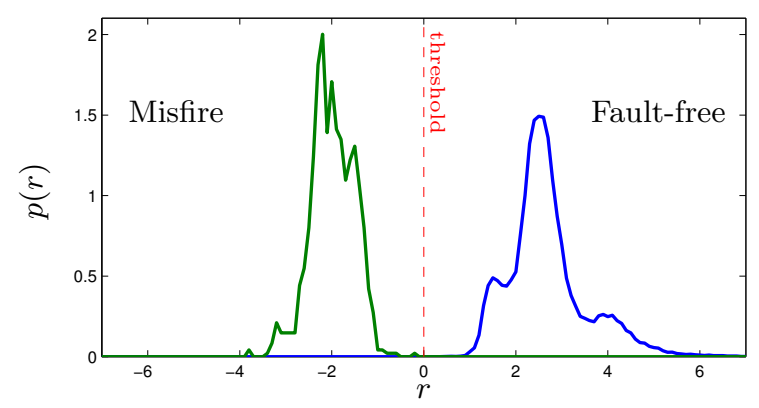

Fig. 26. Histograms of the test quantity $r$ of fault-free data and misfire data given data set 6 when parameters are optimized using SVM.

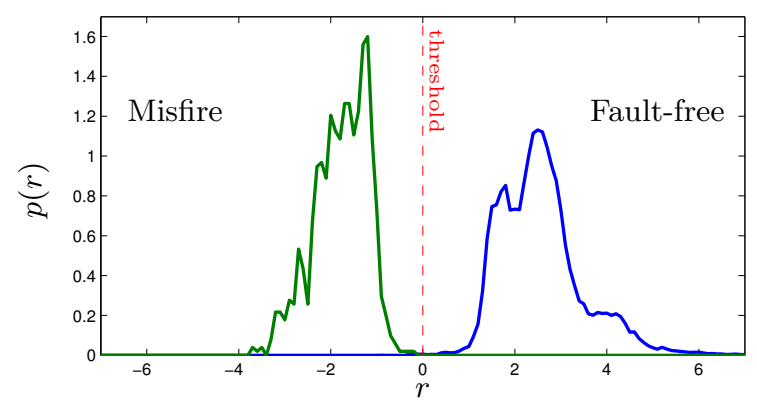

Fig. 27. Histograms of the test quantity $r$ of fault-free data and misfire data given data set 7 when parameters are optimized using SVM.

Table 6

Evaluation of misfire algorithm during cold starts.

\begin{tabular}{cccccc}
\hline Vehicle & Set & Fault-free & Misfire & FA & MD \\
\hline 3 & 12 & 3960 & 82 & $0.35 \%$ & $0 \%$ \\
4 & 13 & 2502 & 52 & $0 \%$ & $0 \%$ \\
4 & 14 & 2716 & 57 & $0.26 \%$ & $3.5 \%$ \\
\hline
\end{tabular}

results show good detection performance. When analyzing the histograms of $r$ for data set 12 in Fig. 28 it is shown that most of the fault-free samples are well separated from the misfires. The number of false alarms in data sets 12 and 14 can be reduced by changing $\beta$ at the cost of increased missed detection rate. 


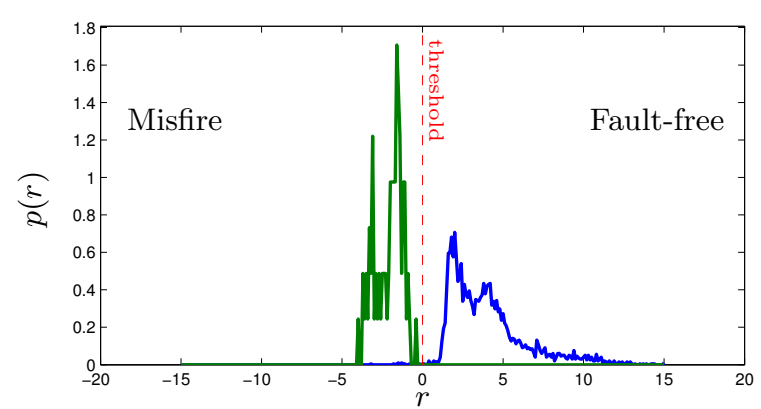

Fig. 28. Histograms of the test quantity $r$ of fault-free data and misfire data given data set 12 when parameters are optimized using SVM.

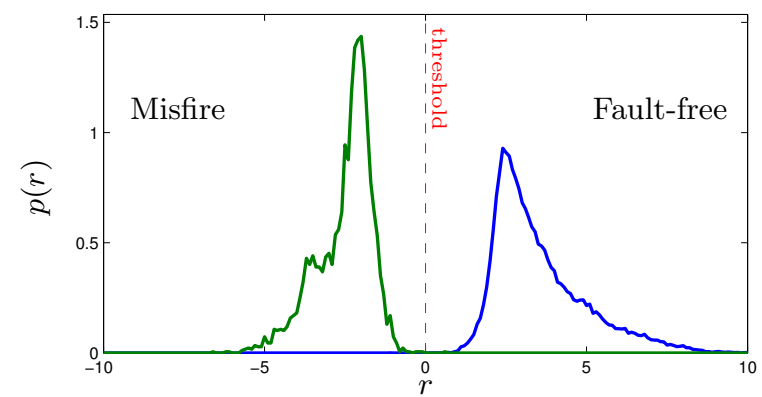

Fig. 29. Histograms of the test quantity $r$ of fault-free data and misfire data from a vehicle with a four cylinder engine.

\subsection{Evaluation using data from a four cylinder engine}

To show that the proposed method can be used for different engine types, data from a vehicle with a four cylinder engine has been used. The measurements have the same resolution as for the six cylinder engine, i.e., $30^{\circ}$. However, the number of samples related to each combustion is six instead of four as for the six cylinder case. The only additional work that needs to be done before implementation is to identify the samples and crank angle counts related to each of the four cylinders. The algorithm is then calibrated using training data and then evaluated using validation data where the result is shown in Fig. 29. The results show that good performance of the misfire detection algorithm is achieved also when implemented for other engine types.

\subsection{Performance analysis}

The evaluation shows that the misfire detection algorithm has an overall good performance with few misclassifications. Here, the performance of the misfire detection algorithm when calibrated, using all available data, will be compared with the results from the analysis performed in Sections 4.4 and 5.1.

In Fig. 30 and Fig 31, histograms estimating the pdfs of fault-free data and misfire data of the test quantity $r$ are

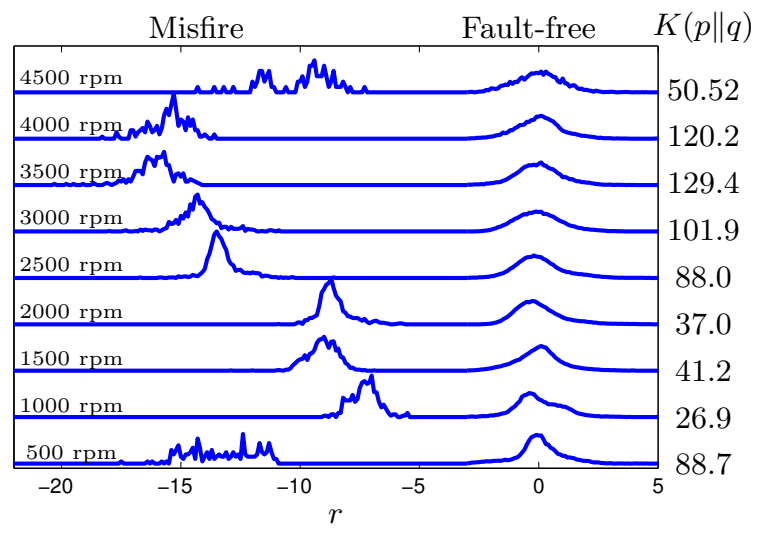

Fig. 30. Histograms of the test quantity $r$ for data from cylinder 1 at the different operating points and computed Kullback-Leibler divergence.

compared for the different operating points for cylinder 1 and 6 respectively together with computed KullbackLeibler divergence. Each curve are histograms representing the pdfs of fault-free data and misfire data from each speed operating point and the Kullback-Leibler divergence is shown to the right. One of the main results from the analysis was that detection performance increased with increasing speed which is also visible when evaluating the misfire detection algorithm. The performance drop at the highest speed operating point can be explained by that all samples from speeds higher than 4250 rpm are all associated to the same operating point of the algorithm. This performance drop can be dealt with by adding more operating points for higher speeds. The computed Kullback-Leibler divergences here are lower compared to the analysis in Section 5, which is probably due to the dimension reduction of the analyzed data from four dimensions to one.

The samples of $r$ at each operating point and cylinder are normalized such that fault-free data has variance one. The figures show that a higher Kullback-Leibler divergence corresponds to a larger separation between data and thus simplifies misfire detection. By comparing the two figures it is visible that misfire data from cylinder 6 is less separated from fault-free data compared to data from cylinder 1 confirm that it is more difficult to detect misfires of cylinder 6 . This is consistent with the results in Fig. 20 and Fig 21 which show that detection performance is lower for cylinder 6 at high velocities compared to cylinder 1 . The variations in distributions between the different cylinders which are collected from three different vehicles confirm the choice of using different parameters, $\mathbf{w}_{k}$ and $\beta$, for different cylinders.

Another observation is that when comparing at which speeds and loads misclassifications occur. In Fig 32, it is shown that most misclassifications occurs at low loads and speeds and only a few at high speeds. Note also that the false alarms at high speeds occurs at speeds that are 


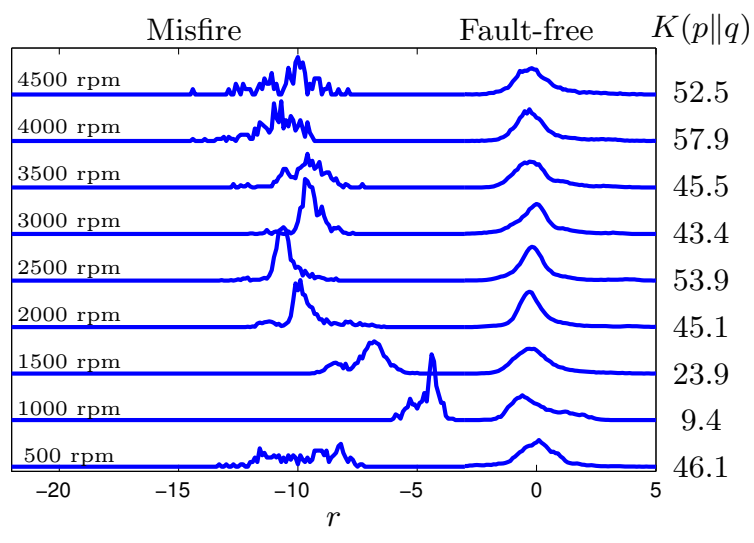

Fig. 31. Histograms of the test quantity $r$ for data from cylinder 6 at the different operating points and computed Kullback-Leibler divergence.

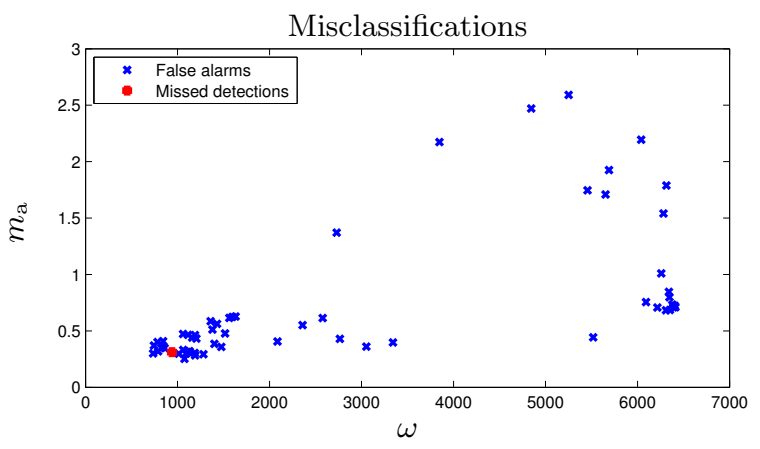

Fig. 32. A plot showing at which speeds and loads misclassifications have occurred during normal driving.

far above the highest speed operating point of the misfire detection algorithm (4500 rpm). Also, there are several misclassifications around speed $1000 \mathrm{rpm}$ and load 0.5. This is consistent with the results seen in Fig. 20 and Fig 21 where the Kullback-Leibler divergence is lowest around that value. Increasing the number of operating points in the algorithm around $1000 \mathrm{rpm}$ and at higher speeds could probably reduce the number of misclassifications in these cases.

\section{Conclusions}

A computationally simple model-based misfire detection algorithm is proposed and an automatic tuning method that uses training data. The detection algorithm uses the flywheel angular velocity signal with an angular sample resolution of $30^{\circ}$ to estimate the torque at the flywheel. A diagnosis algorithm is generated by weighting estimated torque related to the firing cylinder and normalized by the load signal to detect misfires. The algorithm parameters are optimized using Support Vector Machines.

An analysis of misfire detection performance using the Kullback-Leibler divergence shows how the performance varies for different operating points and cylinders. The late ignitions and increased variations in estimated torque during cold starts are handled by considering them separately from normal driving and using different intervals of data in the misfire detection algorithm. The performance analysis also verifies that detection performance is improved when normalizing the estimated torque signal with respect to load.

Evaluations show that the misfire detection algorithm achieves good performance with few false alarms and few missed detections even for difficult cases such as cold starts. Further analysis of the misfire detection algorithm shows that the ability to make a correct classification is consistent with the misfire detection analysis based on the Kullback-Leibler divergence.

\section{Acknowledgement}

The authors would like to thank Volvo Car Corporation in Gothenburg, Sweden, and Ph.D. Sasa Trajkovic for helping us with the data collection.

\section{References}

[1] John Auzins, Hasse Johansson, and Jan Nytomt. Iongap sense in misfire detection, knock and engine control. Technical report, SAE Technical Paper, 1995.

[2] Christopher M. Bishop. Pattern Recognition and Machine Learning (Information Science and Statistics). SpringerVerlag New York, Inc., Secaucus, NJ, USA, 2006.

[3] Jinseok Chang, Manshik Kim, and Kyoungdoug Min. Detection of misfire and knock in spark ignition engines by wavelet transform of engine block vibration signals. Measurement Science and Technology, 13(7):1108, 2002.

[4] Francis T. Connolly and Giorgio Rizzoni. Real time estimation of engine torque for the detection of engine misfires. Journal of Dynamic Systems, Measurement, and Control, 116(4):675-686, 1994.

[5] Corinna Cortes and Vladimir Vapnik. Support-vector networks. Machine Learning, 20(3):273-297, 1995.

[6] S.X. Ding. Model-Based Fault Diagnosis Techniques: Design Schemes, Algorithms, and Tools. Springer-Verlag Berlin Heidelberg, 2008.

[7] Daniel Eriksson. Diagnosability analysis and FDI system design for uncertain systems. Technical report, 2013. LiUTEK-LIC-2013:18, Thesis No. 1584.

[8] Daniel Eriksson, Lars Eriksson, Erik Frisk, and Mattias Krysander. Flywheel angular velocity model for misfire and driveline disturbance simulation. In 7th IFAC Symposium on Advances in Automotive Control, Tokyo, Japan, 2013.

[9] Daniel Eriksson, Erik Frisk, and Mattias Krysander. A method for quantitative fault diagnosability analysis of stochastic linear descriptor models. Automatica, 49(6):1591 - 1600, 2013.

[10] Qianwang Fan, Jiang Bian, Haifeng Lu, Sinyu Tong, and Liguang Li. Misfire detection and re-ignition control by ion current signal feedback during cold start in two-stage directinjection engines. International Journal of Engine Research, 15(1):37-47, 2014. 
[11] T. Hastie, R. Tibshirani, and J. H. Friedman. The Elements of Statistical Learning. Springer, July 2003.

[12] S. Helm, M. Kozek, and S. Jakubek. Combustion Torque Estimation and Misfire Detection for Calibration of Combustion Engines by Parametric Kalman Filtering. Industrial Electronics, IEEE Transactions on, 59(11):43264337, Nov 2012.

[13] J.B. Heywood. Internal combustion engine fundamentals. McGraw-Hill series in mechanical engineering. McGraw-Hill, 1988.

[14] U. Kiencke. Engine misfire detection. Control Engineering Practice, 7(2):203 - 208, 1999.

[15] Kullback, S. and Leibler, R. A. On Information and Sufficiency. Ann. Math. Statist., 22(1):79-86, 1951.

[16] Dag Lundström and Stefan Schagerberg. Misfire detection for prechamber si engines using ion-sensing and rotational speed measurements. Technical report, SAE Technical Paper, 2001.

[17] J Mohammadpour, M Franchek, and K Grigoriadis. A survey on diagnostic methods for automotive engines. International Journal of Engine Research, 13(1):41-64, 2012.

[18] J. Molinar-Monterrubio and R. Castro-Linares. Sliding Mode Observer for Internal Combustion Engine Misfire Detection. In Electronics, Robotics and Automotive Mechanics Conference, 200\%. CERMA 2007, pages 620-624, 2007.

[19] Sanjeev Naik. Advanced misfire detection using adaptive signal processing. International Journal of Adaptive Control and Signal Processing, 18(2):181-198, 2004.

[20] Andrew W. Osburn, Theodore M. Kostek, and Matthew A. Franchek. Residual generation and statistical pattern recognition for engine misfire diagnostics. Mechanical Systems and Signal Processing, 20(8):2232 - 2258, 2006.

[21] Fabrizio Ponti. Development of a torsional behavior powertrain model for multiple misfire detection. Journal of engineering for gas turbines and power, 130(2), 2008.

[22] El-Adl Mohammed Aly Rabeih. Torsional vibration analysis of automotive drivelines. $\mathrm{PhD}$ thesis, The University of Leeds, 1997.

[23] MA Rizvi, Syed Sajjad Haider Zaidi, M Amin Akram, and Aamer I Bhatti. Misfire fault detection in SI engine using sliding mode observer. In IECON 2012-38th Annual Conference on IEEE Industrial Electronics Society, pages 5114-5119. IEEE, 2012.

[24] Muddassar Abbas Rizvi, Aamer Iqbal Bhatti, and Qarab Raza Butt. Hybrid model of the gasoline engine for misfire detection. Industrial Electronics, IEEE Transactions on, 58(8):3680-3692, 2011.

[25] Stefan Schagerberg and Tomas McKelvey. Instantaneous Crankshaft Torque Measurements - Modeling and Validation. SAE Technical Paper 2003-01-0713, 2003.

[26] Abhishek Sharma, V. Sugumaran, and S. Babu Devasenapati. Misfire detection in an $\{\mathrm{IC}\}$ engine using vibration signal and decision tree algorithms. Measurement, 50(0):370 - 380, 2014.

[27] M Shelef and R.W McCabe. Twenty-five years after introduction of automotive catalysts: what next? Catalysis Today, 62(1):35 - 50, 2000.

[28] Sugumaran.V, Ramachandran.K.I, and Babu Devasenapati.S. Article: Misfire detection in a spark ignition engine using support vector machines. International Journal of Computer Applications, 5(6):25-29, August 2010. Published By Foundation of Computer Science.
[29] Francisco V. Tinaut, Andrés Melgar, Hannes Laget, and José I. Domínguez. Misfire and compression fault detection through the energy model. Mechanical Systems and Signal Processing, 21(3):1521 - 1535, 2007.

[30] Yunsong Wang and Fulei Chu. Real-time misfire detection via sliding mode observer. Mechanical Systems and Signal Processing, 19(4):900 - 912, 2005.

[31] YY Wang, V Krishnaswami, and G Rizzoni. Event-based estimation of indicated torque for ic engines using slidingmode observers. Control Engineering Practice, 5(8):11231129, 1997. 